\title{
Measurement and Assessment of Child and Adolescent Personality Pathology: Introduction to the Special Issue
}

\author{
Jennifer L. Tackett
}

Published online: 13 October 2010

(C) Springer Science+Business Media, LLC 2010

An understanding of personality pathology in younger populations is severely deficient, due in part to limitations imposed on youth personality disorder (PD) diagnoses in DSM-IV (American Psychiatric Association 2000). The validity of maladaptive personality traits early in life, however, has been increasingly documented (Tackett et al. 2009), suggesting a need for attention to this understudied area of research. The movement toward a better understanding of child and adolescent PDs is reflected in a recent two-part special issue in Development and Psychopathology focusing on precursors and trajectories of youth PD (Cicchetti and Crick 2009). Although the recent surge in interest in this topic is encouraging, it brings with it a need to closely examine our measurement and assessment of these constructs in younger age groups (Clark 2010). The purpose of this special issue was to highlight approaches toward better measurement and assessment of personality pathology in children and adolescents.

\section{Challenges to PD Measurement in Younger Populations}

In introducing this special issue, I briefly review several of the major challenges facing researchers who are interested in early manifestations of personality pathology. These issues are not necessarily unique to younger age groups, but at present they are all particularly important for researchers studying children and adolescents. Further, these points are

\section{J. L. Tackett $(\bowtie)$}

Department of Psychology, University of Toronto,

100 St. George Street,

Toronto, ON M5S 3G3, Canada

e-mail: tackett@psych.utoronto.ca addressed by the interesting papers and provocative commentaries making up this collection.

Multiple Informants and Multiple Measures The challenge of dealing with multiple informants, particularly the prevalence of disagreement among them, is not a new one for developmental psychopathologists (De Los Reyes and Kazdin 2005). Many of the issues that researchers have been examining in other domains of child clinical research will also pose challenges for researchers interested in youth PDs. Measures of child and adolescent psychopathology often evidence low inter-rater agreement (Achenbach et al. 1987; De Los Reyes and Kazdin 2005; Kotler and McMahon 2005). In addition, the topic of multiple informants has a rich history in the study of normal personality, although these literatures have not yet been merged with one another (Tackett 2010). Whereas most work has focused on finding convergence among multiple informants, recent research has suggested that informant discrepancies also hold validity as an independent construct that may predict maladaptation (De Los Reyes et al. 2010; Tackett 2010). The use of selfreport information is far less common in child research, given that self-report data in younger age groups is often difficult to collect and may have limited validity. Limitations of self-report PD measures in adults may also affect selfreports in youth: social desirability and lack of insight may make self-report PD assessment difficult (Kotler and McMahon 2005). Thus, continued use of multiple informants is necessary for understanding child and adolescent personality pathology, along with continued attention to the difficulties in reconciling disagreements among them (Clark 2010; Morey 2010).

The difficulty in obtaining reliable and valid self-report measures in younger age groups has long pushed developmental psychopathology researchers toward the use of 
multiple methods in their research. Many PD researchers are also turning to methods of assessment beyond selfreport questionnaires and interviews (e.g., Kuo and Linehan 2009), offering an important opportunity for merging these literatures. Similar to the prevalent use of multiple informants with younger populations, researchers frequently use multiple measures in investigations of youth personality pathology, as evidenced by the papers in this special issue. These findings highlight the importance of using multiple measures/methods as they may each provide important, nonoverlapping information (Aelterman et al. 2010; Barry and Wallace 2010; Salekin et al. 2010; Tackett and Ostrov 2010).

Developmental Context A number of additional issues related to developmental context will place pressures on child and adolescent PD research. One criticism of applying PD constructs to younger populations is that the substantial changes in child and adolescent development are not easily reconciled with the stability of adult PD constructs (Kotler and McMahon 2005) but this criticism has not held up to empirical scrutiny (e.g., Lynam et al. 2009). Personality pathology appears to be just as stable in childhood and adolescence as in adulthood (Tackett et al. 2009). Another common criticism is that applying PD labels to youth will be stigmatizing, given the belief that PD constructs are stable and intractable to interventions (Cicchetti and Crick 2009; Esterberg et al. 2010; Tackett et al. 2009). On the other hand, recent efforts toward adolescent intervention have been found to reduce symptoms of personality pathology specifically by using an indicated prevention approach, which relies on identification of early risk factors to determine those adolescents who are most vulnerable to developing adult PDs (Chanen et al. 2008). Indeed, identification of early risk factors may be one of the most critical factors in the development and delivery of effective interventions. In addition, although stability is high, a small proportion of youth who meet criteria for a PD diagnosis fail to meet criteria 1 year later (Esterberg et al. 2010). Thus, an adolescent PD diagnosis need not be seen as deterministic or untreatable.

One important consideration when examining personality pathology across the lifespan is the concept of heterotypic continuity, or the idea that the same underlying trait may manifest differently across time (Cicchetti and Crick 2009). A full understanding of the same construct across development will require more flexible thinking about its manifestation. Esterberg and colleagues (2010) provide examples of early correlates for Cluster A PDs that may represent early phenotypic expression of the same underlying PD construct that is observed in adults (Widiger 2010). In addition, a developmental psychopathology framework emphasizes the importance of studying normal and abnormal development alongside one another (Cicchetti and Crick 2009). This approach will be particularly important for understanding child and adolescent personality pathology, as certain PD traits may be somewhat normative at earlier developmental stages such as adolescence (Clark 2010). This is exemplified by Barry and Wallace (2010), who discuss the nature of normative and maladaptive narcissism and the importance in delineating the distinction between the two in adolescence. These authors illustrate the utility in incorporating multiple measures in order to more comprehensively map out normative versus maladaptive components of PD constructs. Similarly, relational aggression has been linked to different forms of PD, particularly borderline PD, but may also represent normative behavior in some children (Tackett and Ostrov 2010).

All such work requires increased attention to the age group under investigation. Studies investigating circumscribed ages or developmental stages may be useful in investigating changing manifestations across time. For example, critical developmental periods for PDs may provide an opportunity for researchers to focus investigations across relevant developmental transitions (Tackett et al. 2009). The critical periods for PD development appear to be linked to changes in the youth's salient interpersonal relationships, which is not surprising given the integral role that interpersonal functioning plays in PD conceptualization. In contrast to standard approaches to research with adults, researchers cannot apply a static conceptualization of PD constructs across different stages of child and adolescent development until the developmental context of these phenomena are better understood.

Defining the Constructs of Interest Much existing work on child and adolescent PDs, as with many childhood disorders, has largely relied on adult conceptualizations of disorder in defining the constructs of interest. This seems a reasonable place for researchers to start, but it is important to investigate the clinical manifestation and construct validity of personality pathology in younger age groups as a new and independent research question as well. There are a number of issues that researchers encounter when trying to apply adult conceptualizations and measurement to younger populations. Some items used in adults will be inapplicable to youth, whereas others may assess behaviors that are actually normative at younger ages (Kotler and McMahon 2005). Even when adult measures and methods are used as the starting point, researchers must be open to expanding our definition of the construct in order to fully explicate its nature in younger groups (Kotler and McMahon 2005; Tackett et al. 2009). Another potential limitation of this approach is that overreliance on adult measures perpetuates potential measurement issues in new research on younger age groups. For example, researchers have criticized a reification of the most popular measure of 
psychopathy, the Psychopathy Checklist-Revised (PCL-R; Hare 2003), as limiting advancements in this area of study (Skeem and Cooke 2010). Yet, most measures of child and adolescent psychopathy were based on the PCL-R (Kotler and McMahon 2005), lending this area of study with younger groups to the same potential pitfalls.

One method researchers can use to address this challenge is inclusion of both top-down measures (originating from adult research and applied to youth) as well as bottom-up measures (empirically derived in younger populations). For example, Aelterman and colleagues (2010) utilized an adult measure of normal personality, an adult measure of personality pathology, as well as a bottom-up measure of youth personality pathology in the same sample. This allows a much more comprehensive coverage of the PD domain than using adult measures alone, further pointing to potential differences in coverage that may highlight developmental differences. Until recently, a comprehensive bottom-up measure of child personality pathology was sorely lacking. The recent development of the Dimensional Personality Symptom Itempool (DIPSI; De Clercq et al. 2006) has filled this gap and now offers researchers an empirically based tool for investigating these constructs at early ages. The DIPSI holds important utility not only for better understanding the domain of personality pathology, but - as illustrated in the paper by De Clercq and colleagues (2010) - also for better understanding other diagnostic categories from a maladaptive trait perspective, reflecting an expansion in our conceptualization of disorder (Morey 2010).

In addition, the use of multiple informants and methods will be necessary to establish construct validity for early manifestations of PD constructs. The addition of new sources of information should also change our conceptualization of the construct over time. For example, relational aggression was initially developed as a primarily female type of aggression, but more recently the gender differences seem to emerge specifically with parent, and not self, informants (Tackett and Ostrov 2010). Such knowledge should lead to modification of the way relational aggression is conceptualized. The use of behavioral criteria to establish predictive validity of PD constructs will not only provide information about the utility of different informants and methods, but point to evidence for construct validity as well (Clark 2010; Morey 2010; Salekin et al. 2010; Tackett and Ostrov 2010).

\section{Future Directions in PD Research in Younger Populations}

One important future direction in this research areaevident from the manuscripts in this issue - is the turn toward a dimensional conceptualization of personality pathology (Clark 2010; Morey 2010; Widiger 2010). Dimensional approaches have been used to study adult PDs for some time now, but recent considerations by the $D S M-5$ work group suggest a more primary role for dimensional PD models in the future. Importantly, dimensional conceptualizations offer better opportunity to bridge work with different populations, including different age groups (Tackett et al. 2009). The commentaries in this issue by Clark, Morey, and Widiger all offer important points of consideration as we move toward a new dimensional classification system. Many of these points are ripe for study with younger age groups, including the relation between PD types versus traits (Clark 2010), the relative value of different levels of analysis (Morey 2010), and the nature of relations between personality pathology and various Axis I disorders (Widiger 2010).

Many of these papers illustrate the potential utility of examining personality not only in terms of higher-order factors, but also lower-order facets (Aelterman et al. 2010; De Clercq et al. 2010; Salekin et al. 2010). The need to attend to facets is illustrated by Salekin and colleagues (2010); who demonstrate that associations at the domain level may conceal more specific associations at the facet level. For example, facets of the same higher-order domain may relate to the construct in opposing directions: two facets of Extraversion, Assertiveness and Warmth, show opposite connections with psychopathy (Salekin et al. 2010). This information is clearly clinically relevant, yet would not be communicated by results looking only at the higher-order domain of Extraversion. One potential challenge this raises is the lack of empirical consensus on the lower-order structure of child personality relative to the higher-order factors (Morey 2010; Tackett 2006). This remains an important area for future research in the structure and measurement of normal personality traits in younger age groups.

Taken together, the papers in this special issue further our understanding of measurement and assessment of personality pathology in childhood and adolescence. Each manuscript, along with the stimulating commentaries, offers new avenues for future research directions. The area of study in child and adolescent personality pathology is a valid domain of inquiry with great importance and clinical utility. There is still much to learn, and researchers in this area will have an opportunity to make vital contributions to our understanding of personality pathology in the coming years.

\section{References}

Achenbach, T. M., McConaughy, S. H., \& Howell, C. T. (1987). Child/adolescent behavioral and emotional problems: implications of cross-informant correlations for situational specificity. Psychological Bulletin, 101, 213-232. 
Aelterman, N., Decuyper, M., \& De Fruyt, F. (2010). Understanding obsessive-compulsive personality disorder in adolescence: a dimensional personality perspective. Journal of Psychopathology and Behavioral Assessment, this issue.

American Psychiatric Association. (2000). Diagnostic and statistical manual of mental disorders (4th ed. text rev). Washington: American Psychiatric Association. text revision.

Barry, C. T., \& Wallace, M. T. (2010). Current considerations in the assessment of youth narcissism: indicators of pathological and normative development. Journal of Psychopathology and Behavioral Assessment, this issue.

Chanen, A. M., Jackson, H. J., McCutcheon, L. K., Jovev, M., Dudgeon, P., Yuen, H. P., et al. (2008). Early intervention for adolescents with borderline personality disorder using cognitive analytic therapy: randomised controlled trial. The British Journal of Psychiatry, 193, 477-484.

Cicchetti, D., \& Crick, N. R. (2009). Editorial: precursors and diverse pathways to personality disorder in children and adolescents. Development and Psychopathology, 21, 683-685.

Clark, L. A. (2010). Emergent issues in assessing personality pathology: illustrations from two studies on adolescent personality and related pathology. Journal of Psychopathology and Behavioral Assessment, this issue.

De Clercq, B., De Fruyt, F., Van Leeuwen, K., \& Mervielde, I. (2006). The structure of maladaptive personality traits in childhood: a step toward an integrative developmental perspective for DSM-V. Journal of Abnormal Psychology, 115, 639-657.

De Clercq, B., Aelterman, N., De Pauw, S., De Bolle, M., Decuyper, M., \& Tackett, J. L. (2010). Delineating childhood autism spectrum symptoms from a maladaptive trait perspective. Journal of Psychopathology and Behavioral Assessment, this issue.

De Los Reyes, A., \& Kazdin, A. E. (2005). Informant discrepancies in the assessment of childhood psychopathology: a critical review, theoretical framework, and recommendations for further study. Psychological Bulletin, 131, 483-509.

De Los Reyes, A., Alfano, C. A., \& Beidel, D. C. (2010). The relations among measurements of informant discrepancies within a multisite trial of treatments for childhood social phobia. Journal of Abnormal Child Psychology, 38, 395-404.

Esterberg, M. L., Goulding, S. M., \& Walker, E. F. (2010). Cluster A personality disorders: schizotypal, schizoid and paranoid person- ality disorders in childhood and adolescence. Journal of Psychopathology and Behavioral Assessment, this issue.

Hare, R. D. (2003). The Hare psychopathy checklist-revised (PCL-R) manual (2nd ed.). Toronto: Multi-Health Systems.

Kotler, J. S., \& McMahon, R. J. (2005). Child psychopathy: theories, measurement, and relations with the development and persistence of conduct problems. Clinical Child and Family Psychology Review, 8, 291-325.

Kuo, J. R., \& Linehan, M. M. (2009). Disentangling emotion processes in borderline personality disorder: physiological and self-reported assessment of biological vulnerability, baseline intensity, and reactivity to emotionally evocative stimuli. Journal of Abnormal Psychology, 118, 531-544.

Lynam, D. R., Charnigo, R., Moffitt, T. E., Raine, A., Loeber, R., \& Stouthamer-Loeber, M. (2009). The stability of psychopathy across adolescence. Development and Psychopathology, 21, 1133-1153.

Morey, L. C. (2010). Personality disorders in childhood and adolescence: conceptual challenges. Journal of Psychopathology and Behavioral Assessment, this issue.

Salekin, R. T., Debus, S. A., \& Barker, E. D. (2010). Adolescent psychopathy and the five factor model: domain and facet analysis. Journal of Psychopathology and Behavioral Assessment, this issue.

Skeem, J. L., \& Cooke, D. J. (2010). Is criminal behavior a central component of psychopathy? Conceptual directions for resolving the debate. Psychological Assessment, 22, 433-445.

Tackett, J. L. (2006). Evaluating models of the personalitypsychopathology relationship in children and adolescents. Clinical Psychology Review, 26, 584-599.

Tackett, J. L. (2010). Parent informants for child personality: Agreement, discrepancies, and clinical utility. Manuscript currently under review.

Tackett, J. L., \& Ostrov, J. M. (2010). Measuring relational aggression in middle childhood in a multi-informant multi-method study. Journal of Psychopathology and Behavioral Assessment, this issue.

Tackett, J. L., Balsis, S., Oltmanns, T. F., \& Krueger, R. F. (2009). A unifying perspective on personality pathology across the lifespan: developmental considerations for DSM-V. Development and Psychopathology, 21, 687-713.

Widiger, T. A. (2010). Cluster A personality symptomatology in youth. Journal of Psychopathology and Behavioral Assessment, this issue. 